\title{
Mobility Management in Cellular Communication Systems Using Fuzzy Systems
}

\author{
J.J. Astrain ${ }^{1}$, J. Villadangos ${ }^{2}$, M. Castillo ${ }^{1}$, J.R. Garitagoitia ${ }^{1}$, and F. Fariña ${ }^{1}$ \\ 1 Dpt. Matemática e Informática \\ 2 Dpt. Automática y Computación \\ Universidad Pública de Navarra \\ Campus de Arrosadía \\ 31006 Pamplona (Spain) \\ josej . astrain@unavarra.es
}

\begin{abstract}
Mobility management in cellular communication systems is needed to guarantee quality of service, and to offer advanced services based on the user location. High mobility of terminals determines a high effort to predict next movement in order to grant a correct transition to the next phone cell. Then a fuzzy method dealing with the problem of determining the propagation path of a mobile terminal is introduced in this paper. Since multi-path fading and attenuation make difficult to determine the position of a terminal, the use of fuzzy symbols to model this situation allows to work better with this imprecise (fuzzy) information. Finally, the use of a fuzzy automaton allows to improve significatively the final recognition rate of the path followed by a mobile terminal.
\end{abstract}

\section{Introduction}

The scenario of this work is a mobile cellular communication system, where mobility of terminals (mobile stations, MS), users and services must be granted and managed.

Mobile communication systems are limited in terms of bandwidth, and they must deal with non-uniform traffic patterns. So, the development of a mobility management model is justified in order to inter-operate different mobile communication networks. The mobility management model considers three tasks: location, mobile stations should be located in order to find them as fast as possible when a new incoming call occurs; directioning, the knowledge about the direction followed by a mobile station during its movement across the cell allows to offer some intelligent services; and handover control.

Handover processes are the transitions between two contiguous cells performed by a mobile station when travelling across a cellular system. Resources allocated by a particular mobile station when it is placed in a cell must be renegotiated with the next arrival cell when travelling across the network, and disposed after the cell transition. In homogeneous networks, the handover process occurs when signal-noise ratio (or another parameter) is below a given level. 
In heterogeneous ones, the handover takes specific parameters of the networks, aspects about quality of service, and user's preferences into account.

In the literature a great variety of algorithms to manage the mobility of users or terminals has been described, the main part of them considering predictive algorithms because in the new communication environments, both resources and services present mobility. A lot of concepts of mobility were first introduced in cellular mobile networks for voice, now data and multimedia is also included. Mobility models classification (used in computing and communication wireless networks) is provided in [3]. Mobility models (either macroscopic or microscopic) are fundamental to design strategies to update location and paging, to manage resources and to plan the whole network. The mobility model influence will increase as the number of service subscribers grows and the size of the network units (cells) decreases.

The progress observed on wireless communications makes it possible to combine a predictive mobility management with auxiliary storage (dependent on the location, caching, and prefetching methods). Mobile computing in wireless communication systems opens an interesting number of applications including quality of service management (QoS) and final user services. Some examples of predictive applications are: a new architecture to browse web pages [6], where the predictive algorithm is based on a learning automaton that attributes the percentage of cache to the adjacent cells to minimize the connection time between servers and stations; the adaptation of the transport protocol TCP to wireless links in handover situations [7], that uses artificial intelligence techniques (learning automaton) to carry out a trajectory prediction algorithm which can relocate datagrams and store them in the caches of the adjacent cells; an estimation and prediction method for the ATM architecture in the wireless domain [10, where a method improving the reliability on the connection and the efficient use of the bandwidth is introduced using matching of patterns with Kalman filters (stability problems); and so on.

The solution here presented is based on the concept of Shadow Cluster that defines dynamically the influence area of the mobile element, getting an interesting prediction of the trajectory of the mobile and therefore of its influence area. This solution follows a dynamic strategy in contrast to a simple periodic updating of the position of the mobile stations. The proposed system includes some intelligence level needed to interact with the quasi-deterministic behaviour of the user profile movements, and the prediction of the random movements.

Sensitivity to deviations from the real path followed by a MS is controlled by means of editing operations [8]. Paths are modelled as strings of symbols that are classified performing an imperfect string matching between the path followed and the dictionary containing the pattern paths existing in the current cell. The number of patterns that must be saved in the user's profile (user dictionary) is reduced, because only the most frequent paths followed by the user and the main paths followed by the rest of users are considered. The degree of resemblance between two patterns is measured in terms of a fuzzy similarity [5] by way of a fuzzy automaton. Fuzzy techniques perform better than statistical ones for 
this problem because in cellular systems, the signal power used to determine the location of a MS are affected by fading, attenuation and multi-path propagation problems. So, location uncertainties due to the signal triangulation are better described and managed using fuzzy techniques. An advantage of this predictive fuzzy method is that it deals with a non limited number of signal propagation errors and its independence from the system architecture.

Future cellular systems will use microcells 4 to answer the high density of calls. The direct consequence of this will be an increment of the number of handoffs/handovers and so algorithms that complete as fast as possible these processes will be required. The fuzzy system here presented represents an alternative to the growth of the offered services and the effective use of the bandwidth compared with natural solutions such as changes in modulation techniques or special codifications. The research referred here shows the open investigation lines in wireless networks and its integration in the current technology.

The rest of the paper is organized as follows: section 2 is devoted to present the mobility management system model used; section 3 introduces the predictive capabilities of the mobility management system model previously presented; section 4 evaluates the results obtained for this technique; finally conclusions and references end the paper.

\section{Mobility Management System Model}

As introduced in 9], mobility modelling, location tracking and trajectory prediction are suitable in order to improve the QoS of those systems. By predicting future locations and speeds of MSs, it is possible to reduce the number of communication dropped and as a consequence, a higher QoS degree. Since it is very difficult to know exactly the mobile location, an approximate pattern matching is suitable.

In [9], movement is split into the regular part of the movement and the random part. The latter part is modelled according to a Markov chain and is described with a state transition matrix of probabilities, but the random character is not fixed completely in any movement. The regular part of movement lets high prediction velocities and easy calculations while the random part associates with a big process power and a low prediction speed. This prediction algorithm works with regular movements, cycles and simple itineraries, and with new patterns. Besides, the algorithm uses information of the previous stage and probabilistic data and physical restrictions to predict the movement. Mobility prediction is easier when user movements are regulars. Because of that, the first step in the predictive mobility management algorithm consists of detecting roaming patterns that must be stored in a database. Once this information is obtained, which allows to build a cell path dictionary containing the most frequent paths followed by the users of a cell when travelling. After that, user's dictionary is built taking into account the regular movements followed by a user across a specific cell. Both, user and cell dictionaries, constitute a hybrid dictionary that is used by an imperfect string matching system to analyze, by terms of edit 
operations, the similarity between the path followed by a MS and all the paths included in that dictionary.

When the MS follows a non regular movement, the path followed is not included into its user dictionary, but the hybrid dictionary also contents the most frequent paths followed by all the users of this cell. So, the only case whenever the system fails is when the movement followed by the MS is not usually followed neither the user, neither the rest of users. At this point, no prediction must be performed, but in order to avoid this circumstance later, the string corresponding to that movement is added to the user dictionary. Such as, next time the movement could be predicted.

MSs can measure seven different pilot signals powers, since each cell of a cellular system is enclosed by six cells. So, a fuzzy symbol including the ownership degree for each cell is built every time that the MS measures that power. During its movement across the network, a MS builds a string of fuzzy symbols (the known locations of the MS) by concatenating those symbols. The strings obtained are matched with the strings contained in the hybrid dictionary, obtaining a similarity measure calculated by a fuzzy automaton [15]. Then, a macroscopic mobility model is obtained, but a microscopic one is also desired. Then, the same concept is applied to a single cell, but now, the cell is split into different divisions. Three different codification schemes to describe the paths are considered. First one considers cell division in non-overlapped rectangular zones, each one uniquely identified by an alphabetic symbol. Second scheme considers sectorial division of a cell, and third one considers a k-connectivity scheme, where eight alphabetic characters represent all the possible movements that the MS can perform (see figure 11). Now, the ownership degrees managed correspond to the different divisions performed for a cell. In [2, codifications are analyzed.
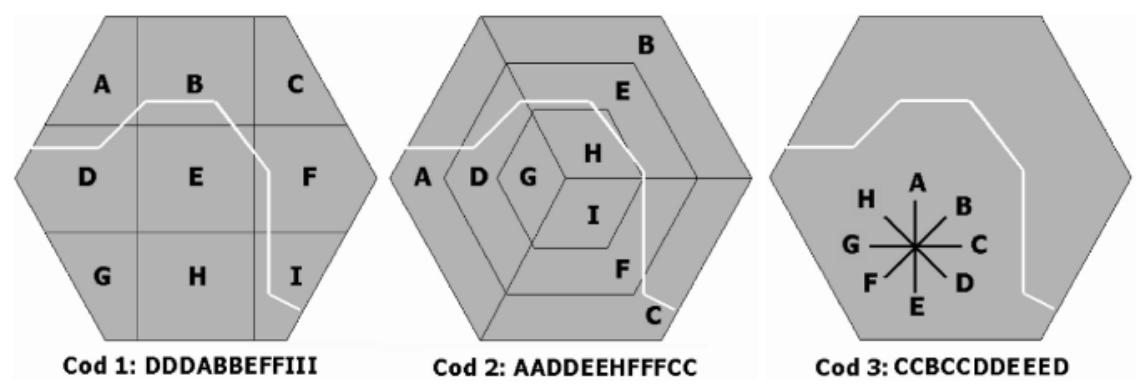

Fig. 1. Path codification schemes.

\section{Trajectory Prediction}

Trajectory prediction can be performed by the MS or by the base station (one BS for each cell of the system). If the prediction is performed by the BS, the storage 
of the dictionaries containing the user and cell more frequently followed paths takes place at the BS side. Furthermore, the BS must predict the movement followed for each user situated on its cell. So, the MS only transmits each fuzzy symbol (pilot signal power measured for each one of the adjacent cells) to its BS associated whenever this measure takes place (each $480 \mathrm{~ms}$ by standard). If the prediction is performed by the MS, dictionaries resides in the own terminal. When the MS is going to access a new cell, the BS of the incoming cell transmits the cell profile (cell dictionary) to the MS. Since the user dictionary is stored in the MS, and the cell dictionary has already been recovered, the MS can perform the calculus of its trajectory itself. So, the MS stores the dictionaries (user and cell profiles), measures the signal power in order to obtain the fuzzy symbol, and calculates the similarity between the string containing the path followed by the MS and the possible paths contained in the hybrid dictionary (more frequent paths of the user and cell dictionaries). In this paper, we have selected this last option, because nowadays mobile phone facilities and resources have considerably grown. Multimedia services require storage and computational resources in the mobile terminals that can be used to implement the fuzzy system here presented. In terms of bytes used, dictionary storage for a cell only requires 10 Kbytes for 50 cell paths.

Each time that the MS calculates the fuzzy symbol $\tilde{\alpha}=$ $\left\{\begin{array}{lllllll}S_{1} & S_{2} & S_{3} & S_{4} & S_{5} & S_{6} & S_{7}\end{array}\right\}$ by measuring the pilot signal power for each one of the adjacent cells, it obtains the existing similarity between the string of fuzzy symbols built by concatenation of the observed symbols and the pattern strings contained in the dictionary. As it can be seen in figure 2 (left), a MS located in cell 1 has 6 adjacent cells, so the given fuzzy symbol has seven components. Signal triangulation (see figure 2(right)) allows the MS to estimate the proximity degree for each cell $\left(S_{i}, \forall i=1,2 \ldots 7\right)$. Since the proximity degree is certainly a fuzzy concept, a fuzzy tool is needed to work with the fuzzy symbol obtained $\tilde{\alpha}$ (composed by these seven proximity degrees). Then, a fuzzy automaton [5] is proposed to deal with the required imperfect string of fuzzy symbols comparison.

\subsection{Formal Trajectory Prediction System}

We propose the use of finite fuzzy automata with empty string transitions $A F F_{\varepsilon}$ $\left(Q, \Sigma, \mu, \mu_{\varepsilon}, \sigma, \eta\right)$ where:

$Q:$ is a finite and non-empty set of states.

$\Sigma$ : is a finite and non-empty set of symbols.

$\mu$ : is a ternary fuzzy relationship over $(Q \times Q \times \Sigma) ; \mu: Q \times Q \times \Sigma \rightarrow[0,1]$. The value $\mu(q, p, x) \in[0,1]$ determines the transition degree from state $q$ to state $p$ by the symbol $x$.

$\mu_{\varepsilon}:$ is a ternary fuzzy relationship over $Q \times Q ; \mu_{\varepsilon}: Q \times Q \rightarrow[0,1]$. The value $\mu_{\varepsilon}(q, p) \in[0,1]$ determines the transition degree from state $q$ to state $p$ without spending any input symbol.

$\sigma$ : is the initial set of fuzzy states; $\sigma \in \mathcal{F}(Q)$. 

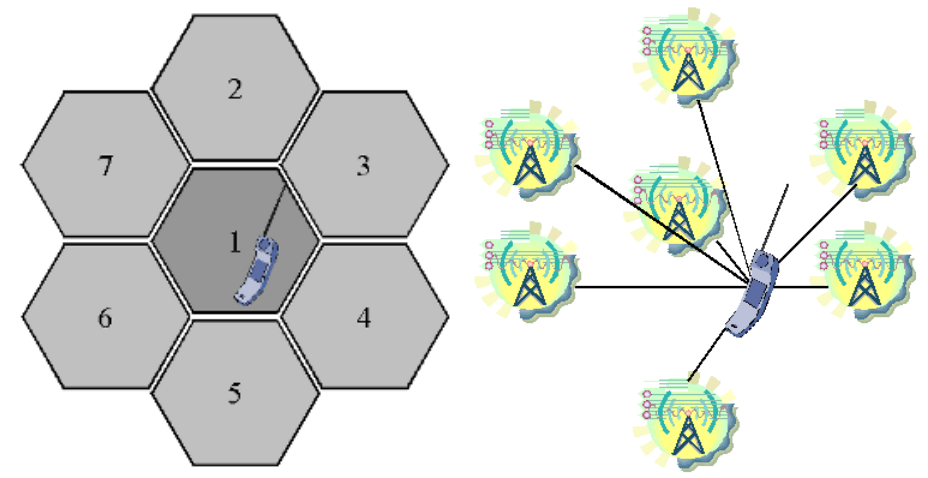

Fig. 2. (left) Cellular communication system. (right) Signal triangulation.

$\eta:$ is the final set of fuzzy states; $\eta \in \mathcal{F}(Q) ; \eta: Q \rightarrow[0,1]$.

The $A F F_{\varepsilon}$ operation over a string $\alpha \in \Sigma^{*}$, is defined by $\left(A F F_{\varepsilon}, T, \hat{\mu}, \hat{\mu}_{\varepsilon}, \mu^{*}\right)$, where:

(i) $A F F_{\varepsilon} \equiv\left(Q, \Sigma, \mu, \mu_{\varepsilon}, \sigma, \eta\right)$ is finite fuzzy automaton with transitions by empty string.

(ii) $T$ is a $t$-norm $T:[0,1]^{2} \rightarrow[0,1]$.

(iii) $\hat{\mu}: \mathcal{F}(Q) \times \Sigma \rightarrow \mathcal{F}(Q)$ is the fuzzy state transition function. Given a fuzzy state $\tilde{Q} \in \mathcal{F}(Q)$ and a symbol $x \in \Sigma, \hat{\mu}(\tilde{Q}, x)$ represents the next reachable fuzzy state. $\hat{\mu}(\tilde{Q}, x)=\tilde{Q} \circ_{T} \mu[x]$ where $\mu[x]$ is the fuzzy binary relation over $Q$ obtained from $\mu$ by the projection over the value $x \in \Sigma$. Then, $\forall p \in Q: \hat{\mu}(\tilde{Q}, x)(p)=\max _{\forall q \in Q}\left\{\mu_{\tilde{Q}}(q) \otimes^{T} \mu(q, p, x)\right\}$.

(iv) $\hat{\mu}_{\varepsilon}: \mathcal{F}(Q) \rightarrow \mathcal{F}(Q)$, is the fuzzy states transition by empty string. Given a fuzzy state $\tilde{Q} \in \mathcal{F}(Q), \hat{\mu}_{\varepsilon}(\tilde{Q})$ represents next reached fuzzy state without consuming an input symbol. $\hat{\mu}_{\varepsilon}(\tilde{Q})=\tilde{Q} \circ_{T} \hat{\mu}_{\varepsilon}^{T}$ where $\hat{\mu}_{\varepsilon}^{T}$ is the $T$-transitive closure of the fuzzy binary relationship $\mu_{\varepsilon} \circ_{T} \hat{\mu}_{\varepsilon}^{T}=\mu_{\varepsilon}^{(n-1) T}$ if $Q$ has cardinality $n$. So, $\forall p \in Q: \hat{\mu}_{\varepsilon}(\tilde{Q})(p)=\max _{\forall q \in Q}\left\{\mu_{\tilde{Q}}(q) \otimes^{T} \hat{\mu}_{\varepsilon}^{T}(q, p)\right\}$.

(v) $\mu^{*}: \mathcal{F}(Q) \times \Sigma^{*} \rightarrow \mathcal{F}(Q)$, is the main transition function for a given string $\alpha \in \Sigma^{*}$ and it is defined by:

a) $\mu^{*}(\tilde{Q}, \varepsilon)=\hat{\mu}_{\varepsilon}(\tilde{Q})=\tilde{Q} \circ_{T} \hat{\mu}_{\varepsilon}^{T}, \forall \tilde{Q} \in \mathcal{F}(\mathcal{Q})$.

b) $\mu^{*}(\tilde{Q}, \alpha x)=\hat{\mu}_{\varepsilon}\left(\hat{\mu}\left(\mu^{*}(\tilde{Q}, \alpha), x\right)\right)=\left(\mu^{*}(\tilde{Q}, \alpha) \circ_{T} \mu[x]\right) \circ_{T} \hat{\mu}_{\varepsilon}^{T}, \forall \alpha \in \Sigma^{*}$, $\forall x \in \Sigma, \forall \tilde{Q} \in \mathcal{F}(\mathcal{Q})$.

As it shows figure 3, the fuzzy automaton is used to compare each string of fuzzy symbols with all the known paths for the current cell. Those paths, stored in terms of symbol strings into a dictionary, can be the paths most frequently followed by a certain user or/and by all the users of this cell. The automaton provides as output the ownership degree of the string of fuzzy symbols to the paths contained in the dictionary. $\frac{D_{1}}{D_{2 \ldots k}}>10^{d d}$ where $k$ is the number of strings (paths) contained in the dictionary, and $d d$ is the decision degree. $d d$ can be 


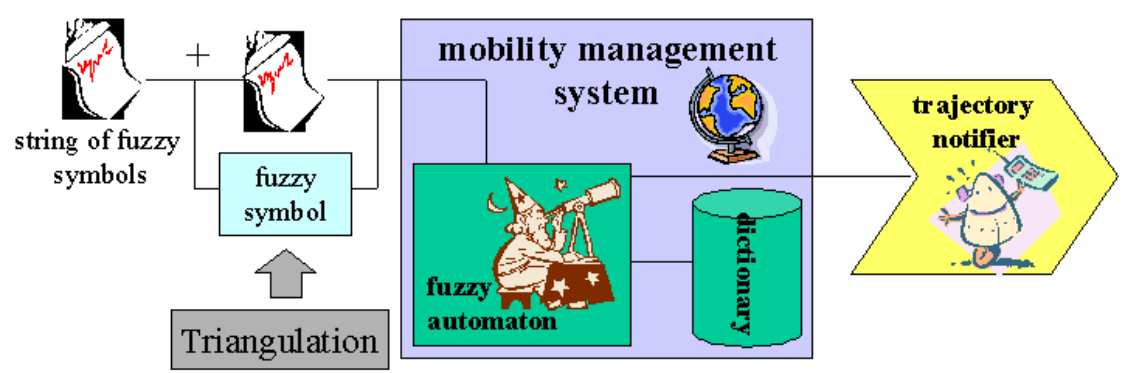

Fig. 3. Mobility management system architecture.

selected in order to increase or reduce the recognition rate, taken into account that a low value for $d d$ can increase the number of false recognitions. A false recognition is the fact to decide that a MS follows a certain path when it does not follow this path. Section 4 explains better the way to select the value of $d d$.

As soon as the MS identifies the movement pattern followed, it notifies the predicted trajectory to the BS. Then, the local BS can contact the BS placed in the destination (incoming) cell. Such as, BS can manage adequately available resources in order to grant quality of service. In the same way, once known the path followed, the MS can receive information about some interesting places or shops placed in its trajectory. It can be seen as a way of sending/receiving selective and dynamic publicity or/and advertisements.

The detection of the trajectory followed does not stop the continuous calculus performed by the MS, because the user can decide to change its movement, and then, prediction must be reformulated. However, the MS only contacts the BS when prediction is performed. Absence of information makes the BS suppose that no prediction has obtained; or if a previous one was formulated, that it has not been modified. Then, the number of signaling messages is considerably reduced, the computation is performed in the MS (client side) and traffic decisions can be taken in advance to the hand-off process.

\section{Robustness of the Mobility Management System Model}

Figure 4 (left) shows the influence of the selected codification in terms of path discrimination. Cuadricular codification needs less (average) time to determine the path followed than other codifications (sectorial and 8-connectivity). Figure 4(right) presents the results obtained when increasing the number of location zones (not available for 8-connectivity codification). The cell is divided in 1, 4, 9, 16 and 25 zones, and we can observe that as the number of zones considered grows, the average time needed to determine the path followed by a Ms inside a cell decreases.

Multiple-classification methods allows to combine the similarity degrees obtained for the three different proposed codifications in order to increase the final recognition rate. Since we are always working with fuzzy symbols and similarity 

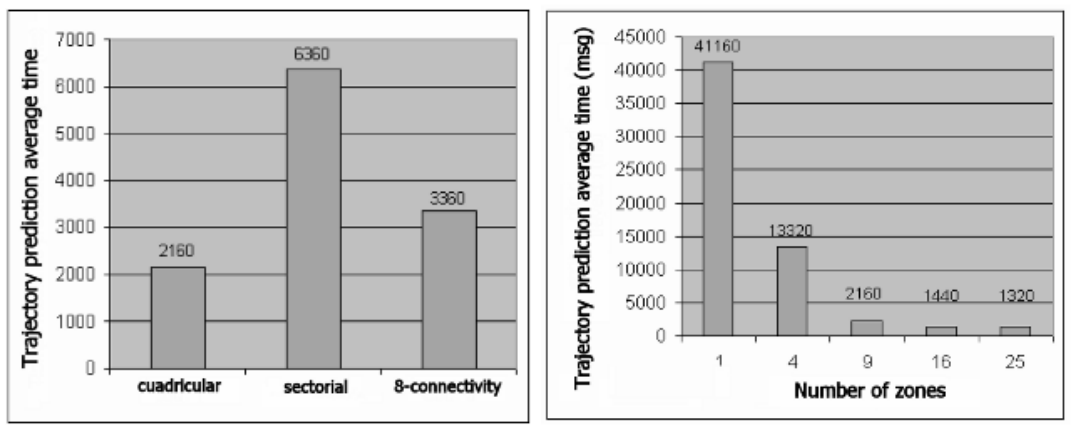

Fig. 4. Trajectory detection average time for different codifications (9 zones) (left) and different number of zones (right).

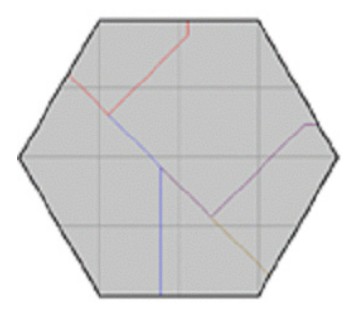

Fig. 5. Most frequent followed trajectories of a cell.

measures, fuzzy inference methods can also be considered to increase the final recognition rate in future works.

In order to evaluate the robustness of the mobility management system model proposed, we have chosen the example described in figure 5 and table 1 .

Table 1. Simulation parameters.

\begin{tabular}{|l|c|}
\hline Cell length $(\mathrm{m})$ & 1000 \\
Location notification time $(\mathrm{ms})$ & 480 \\
Terminal speed $(\mathrm{m} / \mathrm{s})$ & 15 \\
Codification & cuadricular \\
Number of zones & 16 \\
Number of paths included in the dictionary & 4 \\
Average path length $\sharp$ symbols) & 86 \\
Standard deviation path length $(\sharp$ symbols $)$ & 20,4 \\
\hline
\end{tabular}


We have selected a fuzzy automaton using parametric Hamacher $t$-norms and $t$-conorms [5] to predict the trajectories followed by a MS in the related scenario in absence (figure 6) and in presence (figure 7) of signal triangulation errors. The presence of errors is due to the attenuation of the signal and to the multi-path propagation. Attenuation can produces the loss of a symbol, multipath propagation can introduce a new symbol, and both of them can produce a change of two symbols.
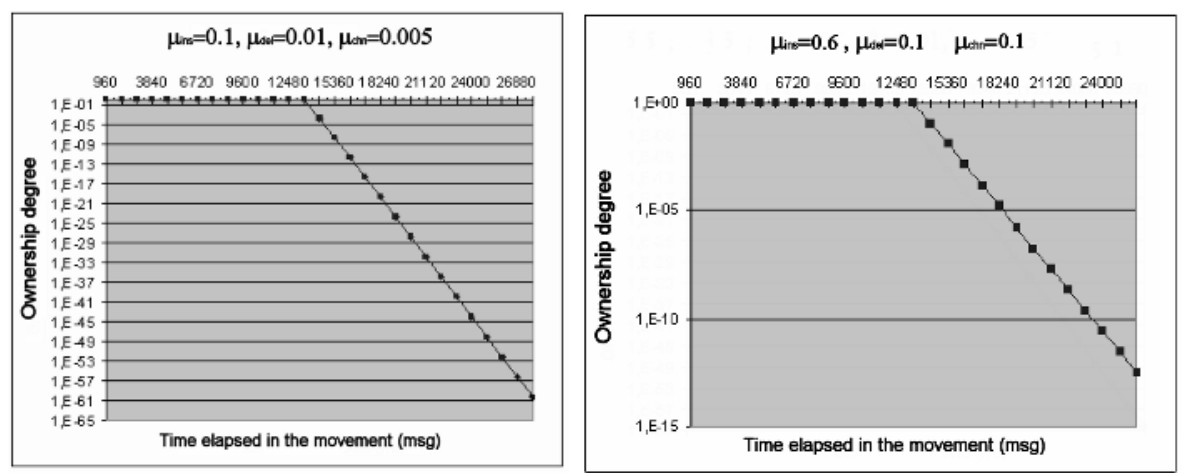

Fig. 6. Ownership degree evolution for the rest of the MS trajectories for different automata configurations (in error absence).
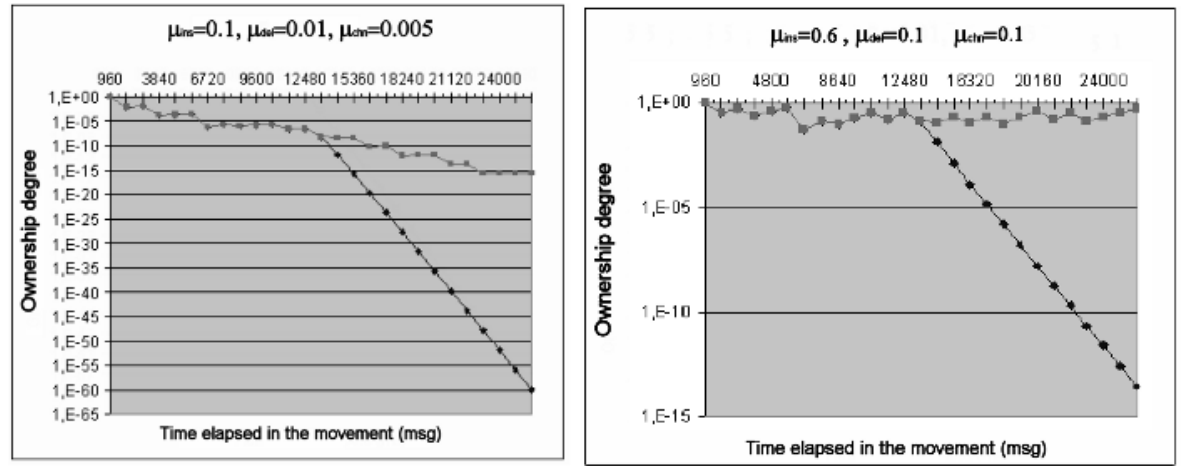

Fig. 7. Ownership degree evolution for the rest of the MS trajectories for different automata configurations (in error presence).

The value of the decision degree $d d$ can be fixed to 5 when the triangulation is error free (non errors are considered) and to 10 when errors in the triangulation are considered. Increasing the value of $d d$ we have a higher certainty in the path estimation, but we need more time to obtain the path estimation. Other values can be selected according to the difficulty of the estimation (number of simi- 
lar/different paths in the cell), the certainty degree required in the estimation, the time required to establish the resources allocation or many other parameters. So, an interesting tuning mechanism to improve the robustness of the mobility management system is introduced.

As figure 8 shows, the mobility of an MS can be represented with a low number of symbols (figure 8 a)) or with a higher number (figure 8 b)). Due to the behaviour of the fuzzy automata, the system can correctly estimate the trajectory followed by the MS in both cases. The automaton deals with insertions, deletion and changes of symbols, so the fact to represent the trajectory followed by the MS with a high number of symbols (high precision degree) is non representative.

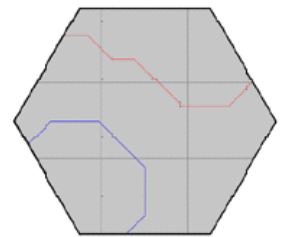

a)

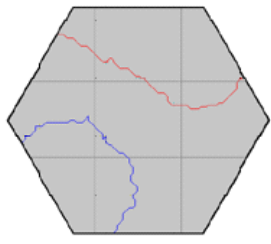

b)

Fig. 8. Mobility representation, different lengths in the string representation.

In order to evaluate the influence of the number of paths considered in a cell, we have defined six different situations illustrated in figure 9. The parameters of the experiment are described in table 2. The number of paths included in the dictionary does not mean an important increase of the time spent by the system prediction, as illustrates figure 10 .

Table 2. Simulation parameters.

\begin{tabular}{|l|c|}
\hline Cell length $(\mathrm{m})$ & 1,000 \\
Location notification time $(\mathrm{ms})$ & 480 \\
Terminal speed $(\mathrm{m} / \mathrm{s})$ & 15 \\
Number of zones (cuadricular and sectorial) & 16 \\
Number of paths included in the dictionary & $2-20$ \\
Average path length $\sharp$ symbols) & 84.37 \\
Standard deviation path length $(\sharp$ symbols) & 30,08 \\
Average path length $(\mathrm{ms})$ & $40,497.6$ \\
Standard deviation path length $(\mathrm{ms})$ & $14,438.35$ \\
\hline
\end{tabular}




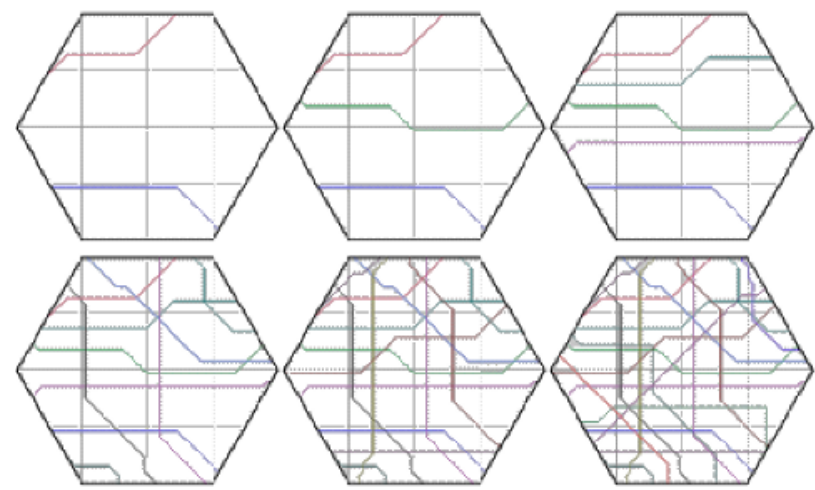

Fig. 9. Different cellscenarios.

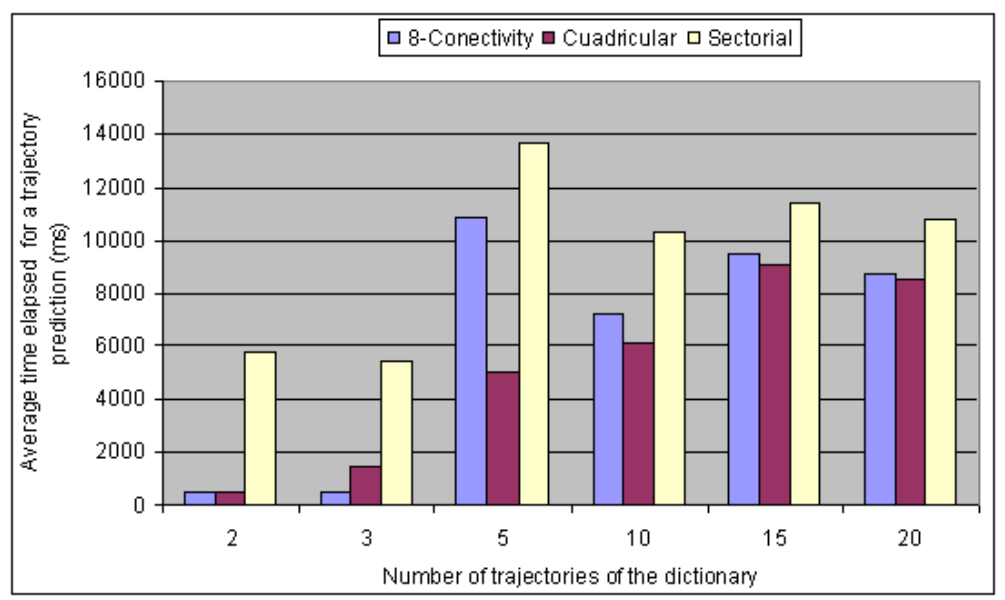

Fig. 10. Influence of the number of paths considered.

Finally, figure 11 presents the average time (measured in milliseconds) needed to estimate the trajectory followed by an MS for the worst case considered in the previous scenario (20 paths). The error rate considered are: 0 , representing that non errors are introduced in the location estimation; $1 / 5$, representing an edition error (insertion, deletion or change) of a symbol each five symbols of the string; and 1/10, representing an edition error each ten symbols of the string. Ten different decision degrees $d d$ are considered in order to evaluate the time needed to ensure a certainty degree in the estimation.

\section{Conclusions}

The new application that is exposed in this article deals with a general problem related to the mobility. Mobility management increases benefits of any mobile 


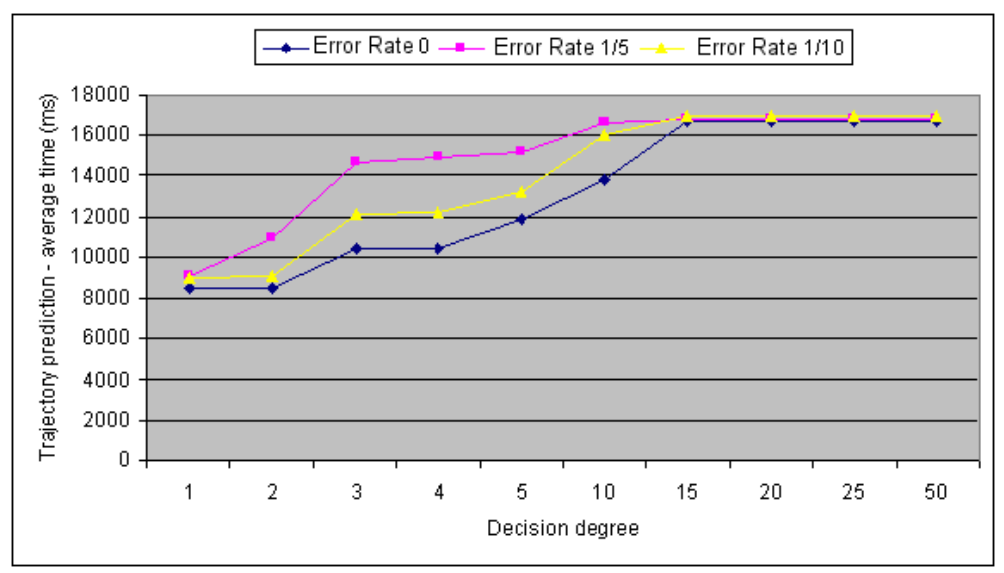

Fig. 11. Average time needed to estimate the trajectory followed by an MS for different decision degrees.

environment. The mobility model introduced is a 2D, microscopic model which can be extended to inter-cell movements and the user can fix its degree of randomness considering the constitutional characteristics.

We propose a fuzzy automaton as a trajectory predictor in order to anticipate the movement of a mobile station in a cellular system. As it has been proved along this work, fuzzy automata allows to manage users mobility in cellular communication systems improving their properties (QoS), and growing the number and quality of services offered by them.

The information harvest, and the decision making is feasible with fuzzy techniques because they handle the existing uncertainties very well, being a really good alternative to other classical statistical techniques.

Three different path codification schemes are considered. Automaton performance for them is evaluated showing that the average time to discriminate the path decrease as the number of zones increase being the rectangular logical division the most interesting codification scheme.

The effect of different fuzzy parameter values for the automaton is analyzed, showing the relevance of them in order to minimize the time needed to discriminate the path followed by the MS among the set of paths contained in the dictionary. In the same way, the automaton works with imperfect strings of symbols due to the vagueness of the location estimation realized by the MS.

Different scenarios and decision degrees are studied in order to show the robustness of the mobility management technique proposed. 


\section{References}

1. J. J. Astrain, J. R. Garitagoitia, J. Villadangos, F. Fariña, A. Córdoba and J. R. González de Mendívil, An Imperfect String matching Experience Using Deformed Fuzzy Automata, Frontiers in Artificial Intelligence and Applications, Soft Computing Systems, vol. 87, pp. 115-123, IOS Press, The Nederlands, Sept. 2002.

2. J. J. Astrain, J. Villadangos, P. J. Menéndez, J. Domínguez, "Improving mobile communication systems QoS by using deformed fuzzy automata", Proceedings of the International Conference in Fuzzy Logic and Technology EUSFLAT 2003, Zittau (Germany), 2003.

3. C. Bettsetter, "Mobility modeling in wireless networks: categorization, smooth movement, and border effects", ACM Mobile Computing and Communications Review, vol. 5, no. 3, pp. 55-67, July 2001.

4. G. Edwards, A. Kandel and R. Sankar, "Fuzzy Handoff Algorithms for Wireless Communication", Fuzzy Sets and Systems, vol. 110, no. 3, pp. 379-388, March 2000.

5. J. R. Garitagoitia, J. R. González de Mendívil, J. Echanobe, J. J. Astrain, and F. Fariña, "Deformed Fuzzy Automata for Correcting Imperfect Strings of Fuzzy Symbols", IEEE Transactions on Fuzzy Systems, vol. 11, no. 3, pp. 299-310, 2003.

6. S. Hadjefthymiades and L. Merakos, "ESW4: Enhanced Scheme for WWW computing in Wireless communication environments", ACM SIGCOMM Computer Communication Review, vol. 29, no. 4, 1999.

7. S. Hadjefthymiades, S. Papayiannis and L. Merakos, "Using path prediction to improve TCP performace in wireless/mobile communications", IEEE Communications Magazine, vol. 40, no. 8, pp. 54-61, Aug. 2002.

8. V. I. Levenshtein, Binary codes capable of correcting deletions, insertions, and reversals, Sov. Phys. Dokl., vol. 10, no. 8, pp. 707-710, 1966.

9. G. Liu and G. Q. Maguire Jr., "A Predictive Mobility Management Scheme for Supporting Wireless Mobile Computing", Technical Report, ITR 95-04, Royal Institute of Technology, Sweden, Jan. 1995.

10. T. Liu, P. Bahl and I. Chlamtac, "Mobility Modeling, Location Tracking, and Trajectory Prediction in Wireless Networks", IEEE JSAC, vol. 16, no. 6, pp. 922936, Aug. 1998. 